\title{
Research and Development on Land Supervision-discovery System Based on Remote Sensing Technologies
}

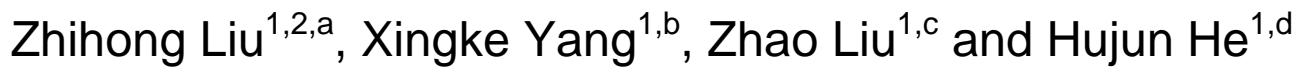 \\ ${ }^{1}$ School of Earth Science and Resources, Chang'an University, Xi'an 710054, China \\ ${ }^{2}$ Xi'an Bureau of State Land Supervision, Xi'an 710075, China \\ ahshengjun@sohu.com, byxk6105@chd.edu.cn, '1298028881@qq.com, ${ }^{d} 513706952 @ q q . c o m$
}

Keywords: Remote Sensing Technologies; Illegal Land Use; Discovery System; Informatization.

\begin{abstract}
Analyzing the existing problems of traditional land law enforcement work and the development significance of land supervision-discovery system based on remote sensing technology, combined with the actual situation of Xi'an Bureau of State Land Supervision, the paper designed land supervision-discovery system based on remote sensing technologies, namely GIS, GPS and RS. The purpose is to regulate the illegal land use and supervise land resources comprehensively using the existing foundation remote sensing data, land use data, the overall planning data of land use and other information resources.
\end{abstract}

\section{Introduction}

Land remote sensing supervision-discovery system is to provide data and information support to supervision work. The purpose is to process, categorize and manage information by classification in order to realize information collection and analysis. This course is based on the satellite remote sensing data and the abnormal information found by the system. In addition, information collected from media exposures, inspections, discourses by citizens, instructions from managers is also important.

Through the development and construction of this system, to realize all the massive remote sensing data, which of Xi 'an Bureau of State Land Supervision(Total area of Xi 'an Bureau is 3.1 million $\mathrm{km}^{2}$, the land area is big, that always has been remote sensing monitoring data and the secondary survey data reproduction, etc.). "The picture of $\mathrm{Xi}$ 'an inspectors" is unified management, and make the establishment of local unified remote sensing which is used "background database" of GIS data. That continuous to update, on the basis of the realize massive remote sensing data and the geographic information of system that browsing fast of GIS data. To Strengthen the remote sensing data and geographic information system (GIS), which results of visual expression and application analysis, through the data mining, and spatial analysis of these results, for these results lay on the foundation for the supervision of business application, for land supervision management to provide the decision support tools.

\section{Collectivity Design}

Three Layer Architecture. The design framework of the software development project, which is based on three layer architecture industry standards. It is a system of flexible structure and easy to adjust, both from the perspective of platform and from the aspects of development. It can be divided into presentation layer, business layer and data layer.

In fact, the traditional sense of the data layer is including data logic layer and physical layer. In this project, in order to reduce the coupling between system function and data, reduce the complex connection between the data itself coupling, and effectively solve the multiple formats/variety of unified management of results of remote sensing data source problem, increased the data model layer, the data are combined with different models in the data model layer. It can meet the different requirements of different management on data management, also defines the relationship between 
the data in the data model, it reduced the complexity of the data, and the amount of data of database construction and maintenance costs, using XML technology to realize data model layer[1-6].

\section{Main Technical Parameters.}

(1)Object-oriented design based on UML

Among the software development technology, the object-oriented software development, become the mainstream technology at present. Object-oriented software engineering method is adopted in this system, including the object-oriented analysis method, the object-oriented modeling, object-oriented programming technique used for the construction and development, in strict accordance with the ideas of software engineering and technical requirements for the project requirement analysis, system design, coding, testing and maintenance, quality control and management and monitoring of the project, the project of each stage are able to provide a complete detailed documentation. At the same time, planning, management, development, follow up and avoid risk of the system construction, must be in strict accordance with the requirements of the software engineering.

In the meanwhile, the software development is a high complexity of knowledge engineering system, software development in accordance with the requirements of software engineering, the system requirements analysis, detailed design, coding and system test phase management program become standardized, submit documents corresponding stage. After all these years the practice of software engineering, software process management has increasingly become the core of the software engineering, the development of this system, combined with the CMM and RUP process management thought control and manage the software development process.

(2) Implement an open mind and integrated solutions

The system as Xi'an Bureau of State Land Supervision application of remote sensing analysis of the results of the integrated development, standards and application forms as well as the purpose of the annual results of remote sensing data are subject to change. And can be expected, with the application of in-depth, in the near future, the system will continue to expand and deepen, in the application of the system architecture and technology portfolio system must ensure that all aspects of application functionality can be easily extended, tightly integrated. Therefore, proposed the thought of open-ended and integration.

In fact, the openness of various application systems integration provides the possibility. Embodied in one integrated system, data and applications on two levels, a large relational database (Oracle/SQLSERVER, etc.) and "data model dynamic organization and drive (data-driven and service)" concept allows the integration of heterogeneous distributed multivariate data truly become a reality. In the system, in the same (or more) a relational database or a computer data file structured data and unstructured data, attribute data, multimedia data, text data, statistical data, and spatial data, etc. and business data centralized storage, by the data model described in XML format, to realize the unified management of data, so as to achieve the purpose of data integration.

Function Modules of the System. The system is developed in accordance with the requirements of the overall design "software architecture map". The system overall design includes results neatening, results management, result inquiry, result analysis, results of application of the several subsystems. They mainly include remote sensing image data browsing, detection of map spot data browsing, map basic operation, map spatial orientation, spatial distance measurement, spatial area measurement, comparative map browsing, figure spot attribute query, summary tables of illegal use and analysis report of law enforcement[6,7].

\section{The Main Function of Land Supervision-discovery System}

\section{The System of General Management Infrastructure.}

\section{(1)Generic Browser framework}

Generic browser frame includes main frame interface, plug-in management, helping management as well as a variety of common functions, the entire main frame includes data model area, viewing area, menu bar, toolbar, status bar and show.

(2)Other features 
User Management Module: it provides configuration, management and licensing of different user privileges, and supporting for multi-user concurrency.

The General Function of the System Framework. Compared to browse: For function of multiphase image browsing and comparative analysis; support the co-browsing of vector data and image; support the not synchronized browsing of vector data and image; support the not synchronized browsing between different image.

Gradual change: Overlay the remote sensing images of more period superposition in the same area, shows the changing process of the same area in the different time through gradual change, has the very good visual effect, convenient to view and analyze the trend of the development of the calendar year in the region.

Flight plug-in: The functions are realized through plug-ins to extend users can custom build flight path, set the corresponding parameters of flight, flight to browse and query the various spatial data on both sides of setting flight path.

Brush plug-in: Users can circle the area of interest when browsing, the data of the brush painting area can be saved, in order to put on record and to check again. At the same time the brush data can be import and export, it can also export the brush data into general GIS data format, for the use of other business.

Results. Including the function of the following aspects: according to the 'standards of consolidated data', for the specification of the naming results to manage the image and vector data by inlay and joint, complete the result.

Production Management. That requiring the following functions: support the importing and exporting of huge amounts of data $(>2.0 \mathrm{G})$; provide the result catalogue and the meta database tool; support the quick query and extracting and browsing the huge amounts of data; it has the function of export or output the interested regional data; it can also download and upload the production from the remote sensing results database.

Results Inquire. The system has the various way of general results query, including the query function provided by the multivariate data management and application platform: administrative region project query; figure spot query; spatial query; attribute query; the raw data query; fuzzy query; regional query; Structured query language query.

Special queries: For instance, by monitoring the city and the way of monitoring year combination query. Or query monitoring data of the present / change situation of land use by city, year and type.

Modules of Data Management and Maintenance. The main content of the module includes the storage and management of spatial data results, data backup and recovery, and the data dictionary maintenance.

Handheld PDA Field Discovery System. PDA handheld field investigation subsystem is to obtain illegal map spots range and corresponding properties, and to achieve convergence with the central data management and processing subsystem. PDA handheld field investigation subsystem includes the following features:

Common basic functions framework: This framework is based on Windows SE system development framework, to achieve the basic operation of remote sensing data.

Spatial data management: Supports the import of remote sensing imagery and vector data multi-period, export, and management functions.

Data exchange module: To achieve conversion of different data formats, center operations data, and handheld PDA industry survey data communication between subsystems.

\section{Conclusion}

The research and development on land supervision-discovery system based on remote sensing technologies can realize the monitor and management of the land in Xi'an rapidly, efficently and safely. The system is not only flexible that users can easily add a plug-in expansion to it, but also compatible that complies with the informationalized construction of land resources overall 
framework. Besides, this system can be easily integrated with others, which is worthy of further research and extension.

\section{References}

[1] XinChen Ning: Management and Application of Remote Sensing Image data Based on Oracle Database, China University of Geosciences, Beijing(2008). (In Chinese)

[2] RuiJun Qi: Research and Practice of Inner Mongolia Land Resource "One Map" Information Engineering, Inner Mongolia Normal University, Huhhot(2012). (In Chinese)

[3] Jinjie Zhang: Research on Planning Support System Based on 3DGIS, China University of Geosciences, Beijing(2009). (In Chinese)

[4] Yingying Li: The application of integration of graphics and information system in land resources field-a case of construction land use auditing information system of Zhejiang province, East China Normal University, Shanghai(2007). (In Chinese)

[5] Yao Sun: The design and research of Kunming Xiaoshao international air port construction engineering geographic information system based on.NET platform, Chengdu University of Technology, Chengdu(2007). (In Chinese)

[6] Yuanyuan Li: Study on the application of the land supervise online system-a case study of Lanzhou land routine supervise, Chang'an University, Xi'an(2012). (In Chinese)

[7] Zhao Liu: The land illegal found system based on remote sensing technology research and development, Chang'an University, Xi'an(2013). (In Chinese) 